\title{
Produção de Membranas Híbridas Zirconizadas de SPEEK/Copolissilsesquioxano para Aplicação em Células a Combustível do tipo PEM
}

\author{
Kelen R. Aguiar, Guilherme P. Batalha, Mariana Peixoto, Airton Ramos, Sérgio H. Pezzin \\ Centro de Ciências Tecnológicas UDESC
}

\begin{abstract}
Resumo: Membranas baseadas em poli(aril éter cetona) sulfonada mostraram ser bastante promissoras para aplicação em células a combustível com membrana trocadora de prótons (PEMFC). O poli(éter-éter-cetona) sulfonado (SPEEK), com elevado grau de sulfonação (GS), apresenta alta condutividade de prótons, mas sofre perda de funcionalidade e condutividade em temperaturas altas e umidades baixas. O desenvolvimento de membranas híbridas é uma das possibilidades para melhorar o desempenho destes materiais. Neste trabalho foram preparadas membranas híbridas zirconizadas de SPEEK/copolissilsesquioxano fosfonado (CF) por casting, a partir de SPEEK com GS entre $60 \%$ e $70 \%$ e soluções de cloreto de zirconila $\left(\mathrm{ZrOCl}_{2}\right) 1,5$, ou $10 \%(\mathrm{~m} / \mathrm{m})$. As membranas foram caracterizadas por espectroscopia na região do infravermelho (FTIR), difratometria de raios-X (DRX), análise termogravimétrica (TG), calorimetria exploratória diferencial (DSC), condutividade de prótons $(\sigma)$ e microscopia eletrônica de varredura (MEV). A análise por energia dispersiva (EDS) confirmou a presença de $\mathrm{Zr}$ em domínios esféricos dispersos homogeneamente pelas membranas, enquanto análises de DRX mostraram que os produtos da zirconização são amorfos. Ensaios de impedância eletroquímica indicam aumento da condutividade protônica com a adição de $\mathrm{CF}$ e 1 ou $5 \%$ de $\mathrm{ZrOCl}_{2}$.
\end{abstract}

Palavras-chave: Células a combustível, membranas trocadoras de prótons, híbridos.

\section{Production of Zirconized SPEEK/Copolysilsesquioxane Hybrid Membranes for Application in Proton Exchange Membrane Fuel Cells}

\begin{abstract}
Membranes based on sulfonated poly(aryl ether ketone) are known to be very promising materials for Proton Exchange Membrane Fuel Cells (PEMFC). Sulfonated poly(ether-ether-ketone) (SPEEK), with high sulfonation degrees (SD), present high proton conductivity. However, they lose functionality and conductivity at high temperatures and low humidity. To enhance the performance of these materials, hybrid membranes have gained considerable attention. In this work, zirconized SPEEK/phosphonated copolysilsesquioxane (CF) hybrid membranes were prepared by casting, from SPEEK $(60 \%<\mathrm{SD}<70 \%)$ and 1, 5, or 10 wt. (\%) zirconyl chloride solutions. The membranes were characterized by infrared spectroscopy (FTIR), X-ray diffractometry (DRX), thermogravimetric analysis (TGA), differential scanning calorimetry (DSC), proton conductivity, and scanning electron microscopy (MEV). Energy-dispersive X-ray spectroscopy (EDS) analyses confirmed the presence of $\mathrm{Zr}$ in spherical domains along the membrane, while DRX showed that the precipitated zirconium compounds had an amorphous character. Electrochemical impedance measurements indicated an increased proton conductivity with the addition of $\mathrm{CF}$ and $\mathrm{ZrOCl}_{2}$.
\end{abstract}

Keywords: Fuel cells, proton exchange membranes, hybrid materials.

\section{Introdução}

Células a combustível com membrana trocadora de prótons (PEMFCs) são consideradas uma das tecnologias de energia limpa mais promissoras e uma fonte adequada de alimentação para aplicações estacionárias e para transporte. Suas maiores vantagens incluem: eficiência acima de $64 \%$, alta densidade de energia e habilidade de operar com combustíveis "limpos" [1]. As duas principais classes de PEMFC incluem as células a combustível de $\mathrm{H}_{2} /$ ar e $\mathrm{H}_{2} / \mathrm{O}_{2}$ e as células diretas de metanol (DMFC's). A atual membrana trocadora de prótons (PEM) de referência para as duas classes de PEMFCs é o $\mathrm{Nafion}^{\circledR}$, um copolímero sulfonado perfluorado ${ }^{[2-4]}$, que exibe boa estabilidade química e também alta condutividade de prótons sob condições hidratadas em temperaturas abaixo de $80{ }^{\circ} \mathrm{C}$. Entretanto, a aplicação destas membranas é limitada devido à perda de condutividade a umidades relativas baixas e temperaturas mais altas. Um esforço generalizado está em andamento para desenvolver membranas poliméricas não perfluoradas mais econômicas para células a combustível. Muitos polímeros termoplásticos aromáticos, tais como poli(aril-éter-cetona)s (por exemplo, PEK, PEEK e
PEKK), poli(éter-sulfona) (PES), polibenzimidazol (PBI), etc., têm excelente estabilidade química, mecânica e termooxidativa, além de custo relativamente baixo ${ }^{[2-7]}$. Membranas baseadas em poli(éter-éter-cetona) sulfonado (SPEEK), por exemplo, mostraram ser muito promissoras para aplicação como PEM. Estas membranas, quando com alto grau de sulfonação (GS > 50\%), mostram uma condutividade de prótons elevada devido à alta absorção de água. Entretanto, esta absorção de água elevada leva a um inchamento excessivo e perda de estabilidade química ${ }^{[8]}$. Por outro lado, é conhecido que a adição de compostos inorgânicos na matriz polimérica aumenta a estabilidade química. De acordo com Kozawa et al. ${ }^{[9]}$, a estabilidade química de membranas de SPEEK/M(HPO $) 2 . \mathrm{nH}_{2} \mathrm{O}$, onde $\mathrm{M}=\mathrm{Sn}, \mathrm{Zr}$ ou Ti, se torna maior com o aumento da interface SPEEK/hidrogenofosfato. Um número considerável de publicações descreve a modificação de membranas de SPEEK com cargas inorgânicas, como fosfato de zircônio $^{[8-11]}$, sílica ${ }^{[12]}$, sílica sulfonada ${ }^{[13]}$, heteropoliácidos ${ }^{[14,15]}$, óxido de zircônio ${ }^{[16]}$ e nanoargilas ${ }^{[17]}$. Essas modificações foram feitas principalmente para suprimir a permeabilidade ao metanol

Autor para correspondência: Sérgio H. Pezzin, Centro de Ciências Tecnológicas, Universidade do Estado de Santa Catarina - UDESC, Rua Paulo Malschitzki, s/n, Campus Universitário Professor Avelino Marcante, CEP 89219-710, Joinville, SC, Brasil, e-mail: pezzin@joinville.udesc.br 
enquanto mantém a condutividade de prótons. Enquanto o óxido de zircônio é usado para reduzir a permeabilidade de água e etanol em membranas, o fosfato de zircônio tem sido usado para auxiliar o transporte de prótons ${ }^{[18]}$. Prado et al. ${ }^{[16]}$ estudaram a dispersão de ácido fosfotúngstico (AFT) e $\mathrm{ZrO}_{2}$ em matriz de poli(éter cetona) (PEK), o que resultou em uma diminuição da condutividade de prótons de $86 \mathrm{mS} . \mathrm{cm}^{-1}$ na membrana sem $\mathrm{ZrO}_{2}$ para $0,3 \mathrm{mS} . \mathrm{cm}^{-1}$ na membrana com $\mathrm{ZrO}_{2}$. Entretanto, a zircônia reduziu o processo de extração do AFT pela água. Alberti et al. ${ }^{[19]}$ sintetizaram sulfofenilfosfonato fosfato de titânio (SFFTi) e utilizaram como carga em Nafion ${ }^{\circledR} 1100$ e observaram um aumento na condutividade quando utilizaram 5 e $20 \%$ de SFFTi. Com $30 \%$ de fosfato de titânio, houve uma redução na condutividade de prótons e aumento da fragilidade do material, reduzindo o contato interfacial entre o polímero e a carga ou entre as partículas. RodríguezCastellón et al. ${ }^{[20]}$ preparam materiais mesoporosos de fosfato de $\mathrm{Zr}(\mathrm{IV})$ e Ti(IV) na presença de brometo de cetiltrimetilamônio, observando um aumento significativo na condutividade de prótons com o aumento da temperatura. Quando considerada a aplicação em células de hidrogênio, polímeros sulfonados desidratam acima de $100{ }^{\circ} \mathrm{C}$, tendo condutividades protônicas reduzidas em níveis de umidade baixos ${ }^{[21]}$. A introdução de polisilsesquioxanos fosfonados na matriz polimérica para formar uma rede semi-interpenetrante, pode fornecer uma estrutura de condução de prótons adequada ${ }^{[22]}$.

Dentro deste contexto, dietilfosfatoetiltrietoxisilano ( $\mathrm{Ph}$-TEOS) e feniltrietoxisilano (PTES) foram copolimerizados através de reações de hidrólise e condensação. O copolisilsesquioxano fosfonado $(\mathrm{CF})$ foi adicionado à matriz SPEEK com GS entre 60 e $70 \%$, juntamente com 1, 5 ou $10 \%$ de cloreto de zirconila, para se obter, após hidrólise dos grupos dietilfosfonatos, membranas híbridas zirconizadas com uma rede semi-interpenetrante. As membranas foram caracterizadas por espectroscopia na região do infravermelho (FTIR), difratometria de raios-X (DRX), termoanálise (TG/DSC), condutividade de prótons e microscopia eletrônica de varredura (MEV).

\section{Material e Métodos}

\section{Materiais}

Poli(éter-éter-cetona) (PEEK), em pó (VICTREX ${ }^{\circledR}$ PEEK $^{\mathrm{TM}}$, 450PF), foi gentilmente fornecido pela Victrex USA, Inc. O PEEK foi seca sob vácuo por pelo menos 48 horas a $90{ }^{\circ} \mathrm{C}$ antes do uso. Ácido sulfúrico 97\% (Cinética), ácido clorídrico 37\% (Cinética), ácido fosfórico $85 \%$ (Sigma-Aldrich), dietilfosfatoetiltrietoxisilano $95 \%$ (ABCR), feniltrietoxisilano 98\% (Sigma-Aldrich), dimetilsulfóxido (DMSO) (Vetec), etanol absoluto (Vetec), hidróxido de sódio
(Vetec) e cloreto de zirconila octahidratado > 99\% (Fluka) foram utilizados sem purificação prévia.

\section{Síntese da SPEEK}

Poli(éter-éter-cetona) sulfonado com GS entre $60-70 \%$ foi sintetizado de acordo com procedimento proposto por Jiang et al. ${ }^{[23]}$. A reação de sulfonação foi realizada dissolvendo $10 \mathrm{~g}$ de PEEK em $150 \mathrm{~mL}$ de ácido sulfúrico $97 \%$ sob vigorosa agitação a temperatura ambiente por 24 horas. A solução foi então gradualmente precipitada em água deionizada a cerca de $5{ }^{\circ} \mathrm{C}$ sob agitação. O polímero foi lavado e filtrado várias vezes até o $\mathrm{pH}$ da água de lavagem ser maior que 5 e deixado em água deionizada por uma noite. A SPEEK, de coloração amarelada, foi seca em estufa a vácuo por 24 horas a $80{ }^{\circ} \mathrm{C}$.

\section{Síntese do copolisilsesquioxano fosfonado - CF}

Copolisilsesquioxano fosfonado com composição de 50:50 (mol:mol) foi sintetizado a partir de dietilfosfatoetiltrietoxisilano (Ph-TEOS) e feniltrietoxisilano (PTES) através de procedimento descrito por Nieuwenhuyse et al. ${ }^{[24]}$ Os reagentes na razão molar determinada, foram dissolvidos em $30 \mathrm{~mL}$ de etanol absoluto e, sob agitação, foi adicionado $1 \mathrm{~mL}$ de $\mathrm{HCl}\left(1 \mathrm{~mol} . \mathrm{L}^{-1}\right)$ como catalisador. A mistura foi mantida sob agitação por 24 horas. Após este período, o solvente foi removido em rota-evaporador. O copolisilsesquioxano, na forma de um líquido viscoso transparente, foi obtido com os grupos fosfônicos na forma éster (Figura 1).

\section{Produção das membranas zirconizadas de SPEEK/CF}

Primeiramente, $0,05 \mathrm{~g}$ de CF 50/50 ( $\mathrm{mol} / \mathrm{mol})$ e $0,95 \mathrm{~g}$ de SPEEK foram dissolvidos em $8 \mathrm{~mL}$ de DMSO em temperatura ambiente e sob agitação para se obter uma solução final de $10 \%(\mathrm{~m} / \mathrm{m}) \mathrm{CF} / \mathrm{SPEEK}$. A este sistema adicionaram-se 0,01, 0,05 ou $0,1 \mathrm{~g}$ de cloreto de zirconila $\left(\mathrm{ZrOCl}_{2} .8 \mathrm{H}_{2} \mathrm{O}\right)$, correspondendo a concentrações de 1,5 e $10 \%(\mathrm{~m} / \mathrm{m})$, respectivamente. As respectivas massas de $\mathrm{ZrOCl}_{2} .8 \mathrm{H}_{2} \mathrm{O}$ foram dissolvidas em $2 \mathrm{~mL}$ de DMSO e aquecidas a $80{ }^{\circ} \mathrm{C}$ por 30 minutos. A mistura das duas soluções foi mantida sob agitação por 2 horas e, posteriormente, vertida em placa de Teflon ${ }^{\circledR}$ para o casting em chapa de aquecimento a $70{ }^{\circ} \mathrm{C}$. As membranas obtidas foram mergulhadas em solução de $\mathrm{H}_{3} \mathrm{PO}_{4}$ 1,0 M por 24 horas para precipitação in situ de hidrogenofosfato de zircônio, $\mathrm{Zr}\left(\mathrm{HPO}_{4}\right)_{2} \cdot \mathrm{H}_{2} \mathrm{O}$, nas regiões hidrofílicas da membrana. Para remover o $\mathrm{ZrOCl}_{2}$ remanescente, as membranas híbridas foram lavadas várias vezes com água deionizada e depois secas em estufa a vácuo por 24 horas a $80^{\circ} \mathrm{C}$.

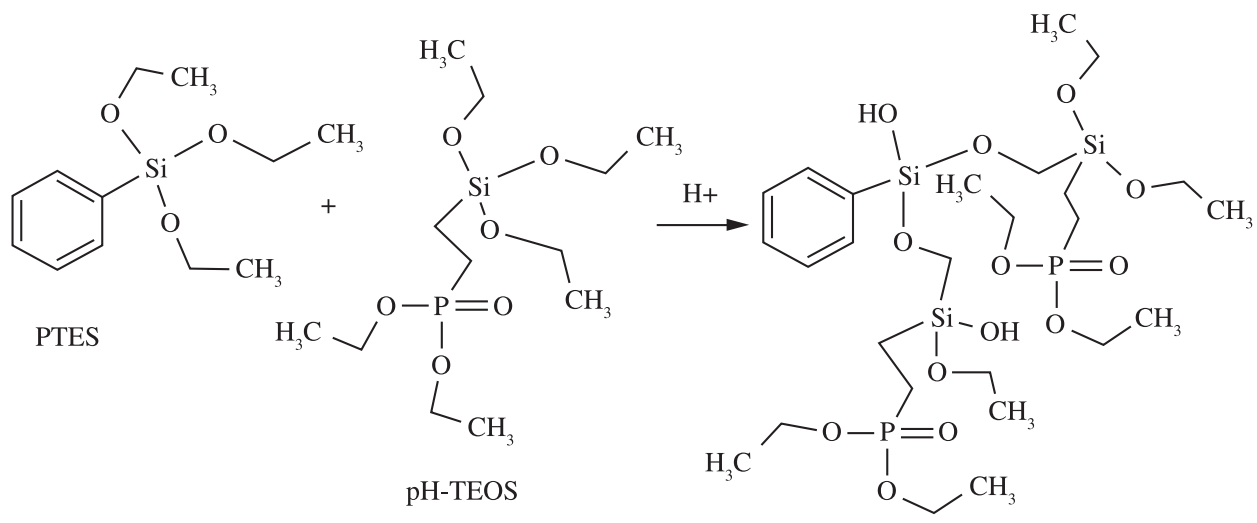

Figura 1. Formação de um trímero durante a síntese do copolisilsesquioxano fosfonado por catálise ácida. 
Hidrólise dos grupos dietilfosfonatos nas membranas zirconizadas de SPEEK/CF

Para a formação do copolissilsesquioxano fosfonado por reações de hidrólise e condensação dos silanos, os grupos fosfônicos presentes no Ph-TEOS devem estar protegidos por grupos etila, os quais devem ser posteriormente removidos para a formação de grupos ácido fosfônico, parcialmente responsáveis pela troca de prótons na membrana. Com este intuito, as membranas foram tratadas com $20 \mathrm{~mL}$ de $\mathrm{HCl} 37 \%$ por 24 horas a $80^{\circ} \mathrm{C}$ a fim de hidrolisar os grupos dietilfosfonatos a grupos ácido fosfônico e promover a formação da rede de copolisilsesquioxano no SPEEK. Após a hidrólise, as membranas foram mantidas em água deionizada por cerca de uma semana antes da caracterização por espectroscopia de impedância.

\section{Caracterizações das membranas}

Os espectros no infravermelho (FTIR) foram coletados usando um espectrofotômetro Perkin-Elmer Spectrum One B, no modo de Refletância Total Atenuada (ATR), sendo realizadas 12 varreduras entre $4000 \mathrm{~cm}^{-1}$ a $650 \mathrm{~cm}^{-1}$ para cada amostra. Os ensaios de análise térmica foram realizados em um equipamento da marca NETZSCH, Júpiter STA 449 C, com sensibilidade de 1,00000 $\mu \mathrm{V} .(\mathrm{mW})^{-1}$. A razão de aquecimento da amostra foi de $10{ }^{\circ} \mathrm{C} / \mathrm{min}$, de $30^{\circ} \mathrm{C}$ até $800{ }^{\circ} \mathrm{C}$, em atmosfera de $\mathrm{N}_{2}$ com vazão de $30 \mathrm{~mL} / \mathrm{min}$. Para a caracterização morfológica das membranas, utilizou-se um microscópio eletrônico de varredura (MEV) da marca ZEISS modelo DSM 940A. A análise semi-quantitativa por energia dispersiva (EDS) foi realizada em um sistema de microsonda Philips PW 2400 acoplado ao MEV. As amostras foram crio-fraturadas em $\mathrm{N}_{2}$ líquido e recobertas com ouro. As análises de DRX foram feitas em equipamento Shimadzu XRD 6000 com fonte de radiação de CuK $\alpha$ operando em $40 \mathrm{kV}$ e $30 \mathrm{~mA}$, a $2 \%$ min em temperatura ambiente. A condutividade de prótons das membranas híbridas foi medida por Espectroscopia de Impedância Eletroquímica (EIS) AC na faixa de frequência de 40-4 × 10 $\mathrm{Hz}$ com tensão de oscilação de $500 \mathrm{mV}$, utilizando um analisador de impedância - Precision Impedance Analyzer 4294A - Agilent. Todas as medidas foram feitas na direção transversal da membrana e tomada a média de 20 varreduras. Cinco membranas de aproximadamente $13 \mathrm{~mm}$ de espessura cada uma, foram presas entre dois eletrodos de aço-inox de $8,75 \mathrm{~mm}$ de diâmetro em uma célula aberta. As medidas foram feitas em temperatura ambiente com membranas hidratadas por pelo menos 24 horas em água deionizada. A principal falha de uma célula aberta é a desidratação do espécime durante a medida. Isso é compensado por vantagens, como fornecer um bom contato eletrodo-espécime (aplicando uma pressão suficiente usando uma carga externa). Ensaios especiais mostraram que espécimes embebidos em água antes do teste e fortemente comprimidas entre os eletrodos, perdem em média $5 \%$ de água durante aquecimento acima de $50^{\circ} \mathrm{C}$ com o tempo. Assim, a real condutividade de prótons pode ser subestimada pela desidratação parcial dos espécimes nessa medida. Portanto, os resultados dos testes de condutividade podem ser considerados como uma avaliação de um limite inferior de condutividade da membrana a uma dada temperatura ${ }^{[14]}$. Nesse trabalho, a condutividade em $40 \mathrm{MHz}$ foi determinada através da equação:

$$
\sigma=\left(\cos \theta_{m} \cdot d\right) /(|Z| \cdot A)
$$

Em que $\theta_{m}$ é o ângulo de fase, $d$ é a distância entre os eletrodos, $|Z|$ é o módulo da impedância e $A$ é a área dos eletrodos.

\section{Discussão dos Resultados}

\section{Síntese do SPEEK e determinação do Grau de Sulfonação (GS)}

O processo de sulfonação do PEEK em ácido sulfúrico envolve um mecanismo de substituição eletrofílica aromática no qual grupos sulfônicos $\left(-\mathrm{SO}_{3} \mathrm{H}\right)$ são introduzidos no segmento hidroquinona da cadeia polimérica, na posição orto $^{[25]}$. O grau de sulfonação para o SPEEK, verificado por análise elementar e FTIR, ficou entre 60 e 70\%. A reação de obtenção de SPEEK é descrita na Figura 2.

Esses grupos ácidos sulfônicos, na presença de água, facilitam o transporte de prótons necessário para alta condutividade nos polímeros. O aumento no número de grupos sulfônicos também aumenta a absorção de água das membranas poliméricas, sendo a sulfonação um método muito eficiente para produzir polímeros condutores de prótons ${ }^{[26]}$. A temperatura de transição vítrea $(\mathrm{Tg})$ também aumenta com o aumento de grupos $-\mathrm{SO}_{3} \mathrm{H}$ na cadeia do PEEK. Este aumento na $\mathrm{Tg}$ reflete em um aumento na associação intermolecular através dos sítios iônicos polares.

Os espectros do PEEK e do SPEEK são mostrados na Figura 3. No espectro de PEEK, a banda mais intensa é a de $1215 \mathrm{~cm}^{-1}$, que corresponde à estrutura de éter aromático, enquanto a banda a $1640 \mathrm{~cm}^{-1}$ corresponde às unidades benzofenona ${ }^{[27]}$. $\mathrm{O}$ espectro FTIR do SPEEK preparado e usado neste trabalho foi muito similar a outros relatados na literatura ${ }^{[28,29]}$, mostrando as bandas de absorção associadas aos grupos ácidos sulfônicos a $3440\left(v \mathrm{O}-\mathrm{H}\right.$ de $\sim \mathrm{SO}_{3} \mathrm{H}$ e água absorvida), $1251\left(v_{\mathrm{as}} \mathrm{O}=\mathrm{S}=\mathrm{O}\right), 1078\left(v_{\mathrm{s}} \mathrm{O}=\mathrm{S}=\mathrm{O}\right), 1021(\mathrm{v} \mathrm{S}=\mathrm{O})$ e $708 \mathrm{~cm}^{-1}$ ( $\left.\vee \mathrm{S}-\mathrm{O}\right)$. A sulfonação também foi confirmada pela divisão da banda de absorção de C-C aromático a $1490 \mathrm{~cm}^{-1}$, com o aparecimento de uma nova banda a $1471 \mathrm{~cm}^{-1}$, e da banda de absorção $\mathrm{a} \sim 1414 \mathrm{~cm}^{-1}$, com uma nova banda a $1402 \mathrm{~cm}^{-1}$. As intensidades relativas das bandas a 1490 e $1471 \mathrm{~cm}^{-1[29]}$ foram consistentes com o grau de sulfonação calculado por análise elementar.

\section{Síntese do Copolissilsesquioxano Fosfonado (CF)}

Nas últimas décadas, diferentes autores relataram a obtenção de cadeias baseadas em silício (siloxanos, silsesquioxanos e silicatos) modificadas com grupos ácidos fosfônicos, por copolimerização de precursores adequados através do processo sol-gel ${ }^{[22,30]}$. Um copolissilsesquioxano 50:50 (mol:mol) com grupos fosfonados ligados covalentemente foi sintetizado neste trabalho utilizando este processo, conforme descrito na Figura 1. A escolha destes silanos precursores teve como objetivo fornecer grupos protogênicos, no caso os grupos fosfônicos $\left(-\mathrm{PO}_{3} \mathrm{H}_{2}\right)$ do Ph-TEOS e favorecer a estabilidade térmica com o uso do PTES, além de explorar a possível interação dos anéis aromáticos do PTES com os anéis aromáticos do SPEEK. A rede inorgânica foi gerada na matriz polimérica pela hidrólise de PTES e Ph-TEOS e condensação para formar ligações siloxano $(\mathrm{Si}-\mathrm{O}-\mathrm{Si})^{[30]}$. O espectro de FTIR do copolisilsesquioxano sintetizado é mostrado na Figura 4.

A absorção relativa ao estiramento assimétrico $\left(v_{\text {ass }}\right)$ da ligação Si$\mathrm{O}-\mathrm{Si}$ pode ser observada na região de $1100-1000 \mathrm{~cm}^{-1}$. No CF obtido,<smiles>CC(C)(O)Oc1cccc(Oc2cccc(C(=O)c3cccc(OC(C)(C)CC4CCCCC4)c3)c2)c1</smiles>

Figura 2. Descrição da reação de sulfonação do PEEK em $\mathrm{H}_{2} \mathrm{SO}_{4}$ concentrado. 
este pico aparece em $1019 \mathrm{~cm}^{-1}$. Também se verificou uma banda em $1086 \mathrm{~cm}^{-1}$ correspondente ao estiramento simétrico $\left(v_{\mathrm{s}}\right)$ da ligação $\mathrm{Si}-\mathrm{O}-\mathrm{C}$, o que mostra que a conversão destas ligações em ligações Si-O-Si não foi completa. A banda em aproximadamente $1050 \mathrm{~cm}^{-1}$ é característica do $v_{\text {ass }}$ da ligação P-O-C. Uma banda intensa em $3212 \mathrm{~cm}^{-1}$ é atribuída à formação de silanóis, água absorvida e etanol gerado durante a condensação. $\mathrm{O}$ espectro da membrana híbrida contendo $10 \%$ de $\mathrm{ZrOCl}_{2}$ é vista na Figura 5. As bandas referentes ao copolisilsesquioxano fosfonado estão presentes neste espectro. Também é possível verificar as bandas de estiramento $v_{(\mathrm{C}=\mathrm{C})}$ do anel aromático em $1594 \mathrm{~cm}^{-1}$, presente tanto na matriz SPEEK quanto no CF. Essa sobreposição de bandas provoca um alargamento na região $1300-1000 \mathrm{~cm}^{-1}$. A banda da carbonila aparece em frequências um pouco mais baixas $\left(1644 \mathrm{~cm}^{-1}\right)$ devido à conjugação com o grupo fenila e a pontes de hidrogênio intermoleculares. Uma nova banda de absorção aparece em $1250 \mathrm{~cm}^{-1}$ correspondente aos grupos

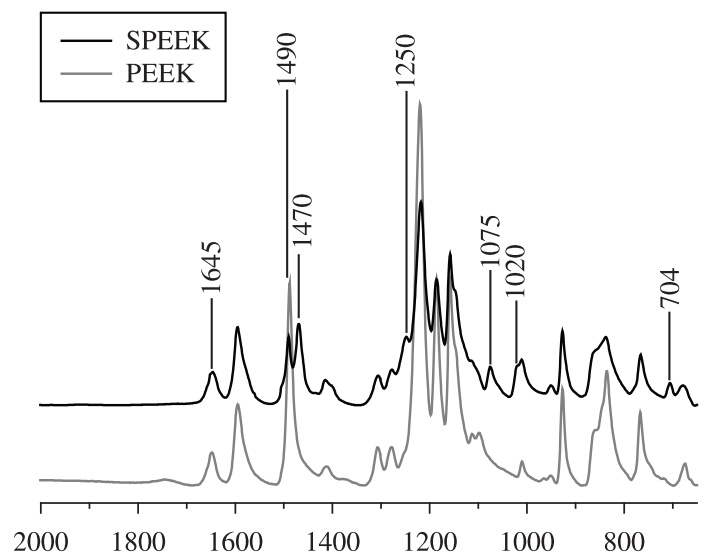

Número de onda $\left(\mathrm{cm}^{-1}\right)$

Figura 3. Espectros de FTIR de PEEK e de SPEEK.

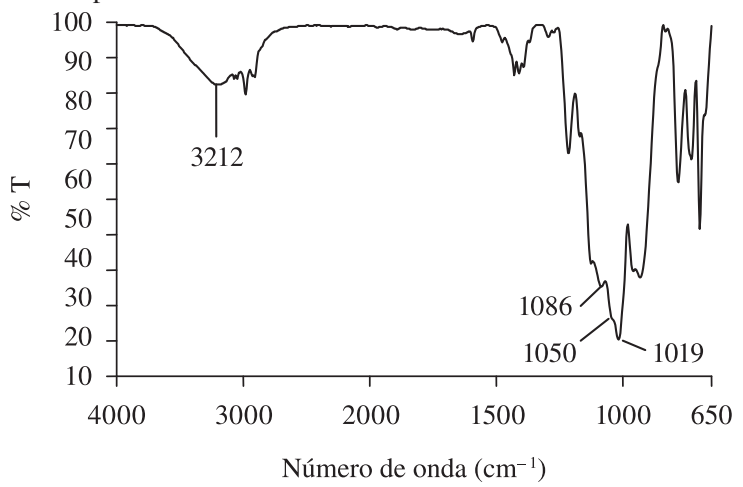

Figura 4. Espectro de FTIR do copolisilsesquioxano fosfonado 50/50 ( $\mathrm{mol} / \mathrm{mol})$.

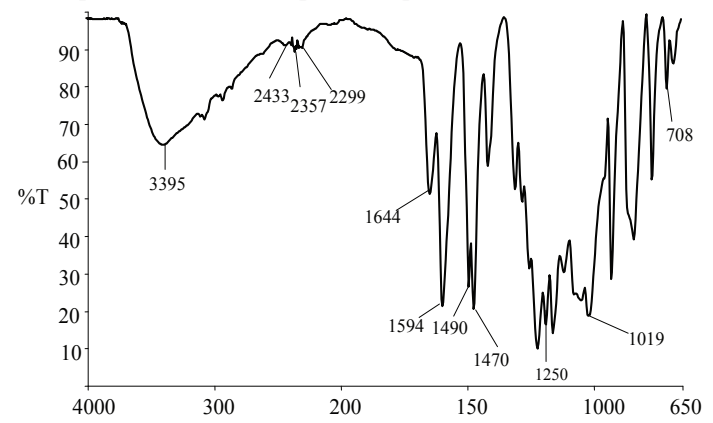

Figura 5. Espectro de FTIR da membrana híbrida SPEEK/CF com $10 \% \mathrm{~m} / \mathrm{m}$ de $\mathrm{ZrOCl}_{2}$. ácidos sulfônicos. Na faixa de $3300-3600 \mathrm{~cm}^{-1}$ ocorre a absorção do $-\mathrm{OH}$ dos grupos $-\mathrm{SO}_{3} \mathrm{H}$ assim como o estiramento $v_{(\mathrm{O}-\mathrm{H})}$ proveniente de pontes de hidrogênio entre os grupos sulfônicos e fosfônicos $\left(\mathrm{SO}_{3}^{-} \mathrm{H}_{2} \mathrm{O}^{+}---0=P\left(\mathrm{OH}_{2}\right)\right)^{[31]}$. Bandas de intensidade semelhante entre 2600-2100 $\mathrm{cm}^{-1}$ são características de ligações hidrogênio dos grupos ácido fosfônicos ${ }^{[32]}$. O estiramento da ligação $v_{(\mathrm{S}-\mathrm{O})}$ aparece em $708 \mathrm{~cm}^{-1}$.

\section{Modificação da matriz SPEEK com cloreto de zirconila e copolissilesquioxano fosfonado}

As membranas produzidas por casting contendo diferentes concentrações de cloreto de zirconila foram tratadas com solução de $\mathrm{H}_{3} \mathrm{PO}_{4} 1 \mathrm{M}$ para promover a zirconização da membrana, com a possível geração in situ de $\mathrm{Zr}\left(\mathrm{HPO}_{4}\right)_{2}(\mathrm{ZrP})$ nos poros hidrofílicos da matriz polimérica SPEEK. O maior intuito deste trabalho foi o de produzir um condutor iônico, sendo importante que o ZrP esteja na forma amorfa e não na forma cristalina lamelar. Enquanto a condutividade do composto cristalino é muito baixa $\left(10^{-7} \mathrm{~S} \mathrm{~cm}^{-1}\right)$, a do material amorfo é muito maior $\left(10^{-3} \mathrm{~S} \mathrm{~cm}^{-1}\right)$ e mais interessante para aplicações em células a combustível ${ }^{[33]}$.

É bem conhecido que precipitados amorfos com composição de $\mathrm{M}(\mathrm{IV})\left(\mathrm{HPO}_{4}\right)_{2-\mathrm{x}}(\mathrm{OH}) 2 \mathrm{x} \cdot \mathrm{nH}_{2} \mathrm{O}$ são facilmente formados pela reação entre um sal de um metal tetravalente ( $\mathrm{Zr}, \mathrm{Ti}, \mathrm{Ce}, \mathrm{Th}, \mathrm{Sn}$, etc...) e uma solução de ácido fosfórico. A síntese do $\mathrm{ZrP}$ se dá pela condensação do cloreto de zirconila e ácido fosfórico através da seguinte reação ${ }^{[34]}$.

$$
\mathrm{ZrOCl}_{2}+2 \mathrm{H}_{3} \mathrm{PO}_{4} \rightarrow \mathrm{Zr}\left(\mathrm{HPO}_{4}\right)_{2}+2 \mathrm{HCl}
$$

A síntese ex-situ foi realizada e obteve-se um gel branco, que após filtrado, lavado com água deionizada e seco apresentou-se como um sólido branco o qual foi caracterizado por DRX.

É importante observar que este trabalho trata de sistemas com silsesquioxanos fosfonados, os quais também podem reagir com o $\mathrm{ZrOCl}_{2}$ para formar complexos com zircônio ${ }^{[33]}$. Após reação dos grupos fosfatos/fosfônicos da superfície da rede de silsesquioxano com $\mathrm{ZrOCl}_{2(\mathrm{aq})}$, os fosfatos ligados à rede de sílica formam um complexo com $\mathrm{Zr}^{++}$na forma de [ $\mathrm{SiOPO}_{3} \mathrm{ZrX}_{2}$ ]. Normalmente a formação deste complexo é verificada por RMN de ${ }^{31} \mathrm{P}^{[33,35]}$ já que a estreita ressonância associada ao $\mathrm{SiOPO}_{3} \mathrm{H}_{2}$ a $\delta=-11.8 \mathrm{ppm}$ alarga quando ocorre a complexação com $\mathrm{Zr}^{4+}$ e duas outras ressonâncias são observadas a -14.8 ppm e -19.1 ppm. Kohli e Blanchard ${ }^{[35]}$ relataram a formação de $\mathrm{Zr}\left(\mathrm{HPO}_{4}\right)_{2}$ por tratamento de redes de sílica fosfonada com $\mathrm{ZrOCl}_{2}$ por $\mathrm{RMN} \mathrm{de}{ }^{31} \mathrm{P}$, sendo que, devido à morfologia superficial da rede de sílica, ainda pode existir fosfato não complexado $\left(\mathrm{SiOPO}_{3} \mathrm{H}_{2}\right)$ e $\mathrm{H}_{3} \mathrm{PO}_{4}$ adsorvido após a zirconação. Este material é diferente do $\alpha-\mathrm{Zr}\left(\mathrm{HPO}_{4}\right)_{2}$, cristalino e lamelar, que pode ser produzido pela reação entre $\mathrm{ZrO}_{2} \mathrm{e}$ $\mathrm{H}_{3} \mathrm{PO}_{4}$ em um solvente adequado ${ }^{[18]}$.

\section{Difratometria de Raios-X (DRX) e Microscopia Eletrônica de Varredura (MEV)}

O difratograma do SPEEK com grau intermediário de sulfonação (Figura 6) mostra somente um pico amorfo com intensidade máxima em $2 \theta=10-30^{\circ}$, estando de acordo com o observado por Zaidi ${ }^{[14]}$. A introdução de grupos $\mathrm{SO}_{3} \mathrm{H}$ em PEEK altera a conformação e empacotamento da cadeia levando a perda de cristalinidade. $\mathrm{O}$ difratograma do PEEK (não mostrado aqui) mostra quatro picos cristalinos intensos em $2 \theta=20^{\circ}, 21^{\circ}, 23^{\circ}$, e $29^{\circ}$ e um halo amorfo por volta de $20^{\circ[36]}$. Esses picos correspondem respectivamente à reflexões nos planos 100, 111, 200 e 211 $1^{[37]}$. Os difratogramas da membrana híbrida tratada com $1 \% \mathrm{ZrOCl}_{2}$, do $\mathrm{ZrP}$ sintetizado ex-situ, da SPEEK e do $\mathrm{ZrOCl}_{2} .8 \mathrm{H}_{2} \mathrm{O}$ estão reunidos na Figura 6. Verifica-se que os picos da estrutura cristalina do $\mathrm{ZrOCl}_{2} \cdot 8 \mathrm{H}_{2} \mathrm{O}$ desaparecem após a incorporação à membrana e a 
reação com $\mathrm{H}_{3} \mathrm{PO}_{4}$, sendo que todos os produtos sintetizados neste trabalho apresentaram estrutura amorfa.

A Figura 7 mostra as micrografias eletrônicas de varredura da região da fratura da membrana híbrida. Na ampliação da Figura $7 \mathrm{~b}$ é possível observar que a adesão entre os domínios inorgânicos de $\mathrm{ZrP}$ e a matriz polimérica é baixa e cavidades estão presentes. No interior destas cavidades pode-se visualizar a presença de partículas precipitadas de $\mathrm{ZrP}$ com dimensões de aproximadamente $400 \mathrm{~nm}$. Na Figura 7c, observou-se que não houve a formação de cavidades e a adesão entre as fases aumentou. A morfologia apresentada pela membrana com $10 \%$ de $\mathrm{ZrOCl}_{2}$ na Figura $7 \mathrm{c}$, revela uma estrutura heterogênea onde o contato entre a membrana e os eletrodos pode ficar prejudicado, interferindo nos resultados de condutividade de prótons.

A análise por EDS mostrou uma dispersão homogênea do Zr na matriz SPEEK, apresentando valores de aproximadamente $6 \%$ de $\mathrm{Zr}$ incorporado na membrana em duas regiões distintas. A microanálise da Figura 8 confirma a presença de ZrP na membrana híbrida.

\section{Condutividade de prótons}

Para as PEMs, a condutividade de prótons da membrana é particularmente importante, uma vez que tem um papel significativo no desempenho da célula combustível. As membranas foram analisadas usando espectroscopia de impedância eletroquímica (EIS). Os resultados de condutividade, obtidos em temperatura

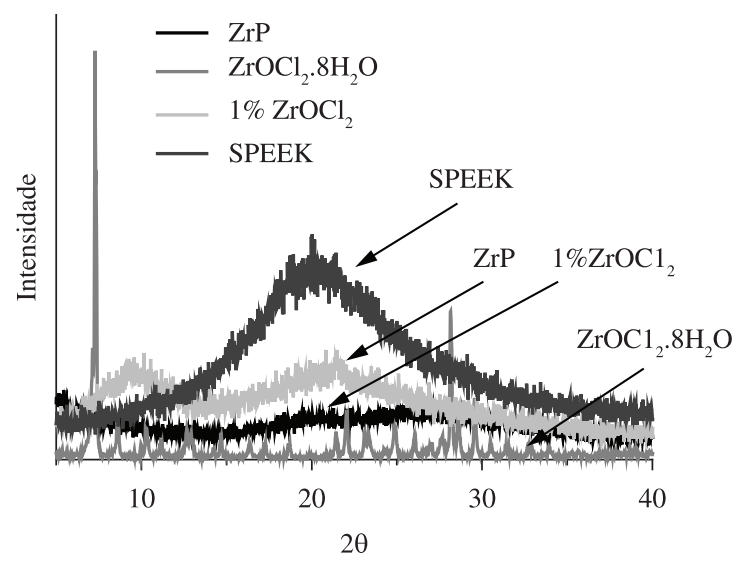

Figura 6. Difratogramas do $\mathrm{ZrOCl}_{2} \cdot 8 \mathrm{H}_{2} \mathrm{O}$, da membrana híbrida preparada com solução $1 \%$ de $\mathrm{ZrOCl}_{2}$, do SPEEK e de $\mathrm{ZrP}$ sintetizado ex-situ. ambiente das membranas hidratadas e retiradas na frequência máxima de trabalho (40 MHz), estão representadas na Figura 9.

$\mathrm{Na}$ Figura 9 observa-se que quando adicionados $1 \%$ e $5 \%$ de $\mathrm{ZrOCl}_{2}$ à matriz híbrida $\mathrm{SPEEK} / \mathrm{CF}$, a condutividade das membranas aumenta de 2,44 para 5,0 e 5,91 mS. $\mathrm{cm}^{-1}$, respectivamente. Com $10 \%$ há uma redução na $\sigma$ quando comparada aos outros teores de $\mathrm{ZrOCl}_{2}$, porém esta continua sendo maior que a da matriz SPEEK. Neste caso, a formação de ZrP no polímero sulfonado pode levar à formação de ligações $\mathrm{Zr}-\mathrm{O}-\mathrm{SO}_{2}$, o que representa não somente a complexação do zircônio com os grupos sulfônicos, mas a desativação parcial dos sítios condutores de prótons ${ }^{[11]}$. Uma estrutura esquemática para a possível ligação entre $\mathrm{ZrP}$ e grupos - $\mathrm{SO}_{3} \mathrm{H}$ da matriz polimérica está representada na Figura 10.

O ZrP precipitado nos poros da membrana forma depósitos, os quais podem bloquear a passagem dos íons através dos canais, podendo contribuir com a redução da condutividade em teores elevados de ZrP. A condutividade pode ser atribuída mais ao $\mathrm{ZrP}$ amorfo, que segundo a literatura possui uma condutividade de prótons variando entre $1-5 \mathrm{mS} . \mathrm{cm}^{-1}$, que aos próprios grupos $-\mathrm{SO}_{3} \mathrm{H}^{[30]}$. Além disso, a hidrólise dos grupos dietilfosfonatos do CF contribuem para a formação de uma estrutura de rede semi-interpenetrante adequada para a transferência de prótons ${ }^{[22]}$.

\section{Análise térmica}

Os termogramas do SPEEK e das membranas híbridas de SPEEK/CF zirconizadas com 1,5 ou $10 \%$ de $\mathrm{ZrOCl}_{2}$ estão representadas na Figura 11. Todas as curvas das diferentes composições apresentaram três estágios de perda de massa. O primeiro, abaixo de $200{ }^{\circ} \mathrm{C}$ corresponde à evaporação de solvente (DMSO), água e etanol formado pela pós-condensação do copolissilsesquioxano. Para as membranas híbridas, esse estágio de perda de massa ocorre em temperaturas ligeiramente maiores que para SPEEK, devido ao maior número de moléculas de água associadas aos grupos funcionais $-\mathrm{PO}_{3} \mathrm{He}-\mathrm{SO}_{3} \mathrm{H}$. $\mathrm{O}$ segundo estágio de perda de massa é referente à decomposição dos grupos $\mathrm{SO}_{3} \mathrm{H}$ do $\mathrm{SPEEK}$ e se dá entre $200-220{ }^{\circ} \mathrm{C}$. Para as membranas com adição de $1 \%$ e $5 \%$ de cloreto de zirconila, a dessulfonação inicia por volta dos $300{ }^{\circ} \mathrm{C}$. Para aquela obtida com a adição de $\mathrm{ZrOCl}_{2} 10 \%$, a degradação dos grupos sulfônicos ocorre a aproximadamente $320^{\circ} \mathrm{C}$. A degradação da cadeia principal do PEEK ocorre por volta de $490{ }^{\circ} \mathrm{C}$ e para as demais membranas, entre $480-630{ }^{\circ} \mathrm{C}^{[39]}$. É interessante observar que a decomposição dos grupos sulfônicos foi retardada (220-300 ㄷ) devido à zirconização da matriz SPEEK. Isso é explicado

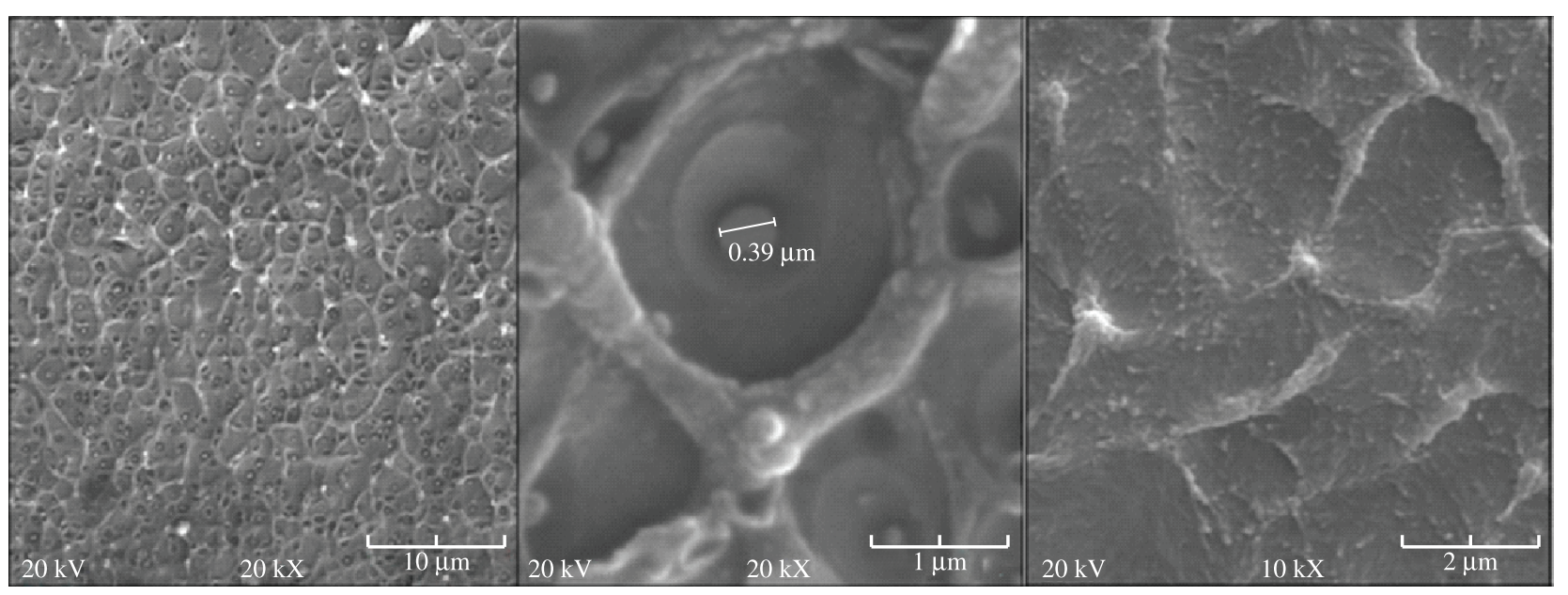

(a)

(b)

(c)

Figura 7. Micrografias da região de fratura da membrana híbrida: a) preparada com $1 \%(\mathrm{~m} / \mathrm{m})$ de $\mathrm{ZrOCl}_{2}$; b) ampliação de uma cavidade; c) micrografia da região da fratura da membrana preparada com $10 \%(\mathrm{~m} / \mathrm{m})$ de $\mathrm{ZrOCl}_{2}$. 
pela formação do complexo entre SPEEK e ZrP, mostrado na Figura 9. Esse método resultou, portanto, na formação de membranas híbridas mais resistentes à degradação térmica.

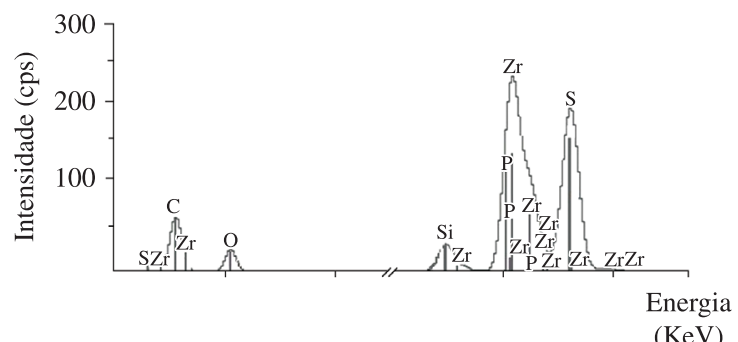

Figura 8. Espectro EDS de membrana SPEEK/CF preparada com $1 \%(\mathrm{~m} / \mathrm{m})$ de $\mathrm{ZrOCl}_{2}$.

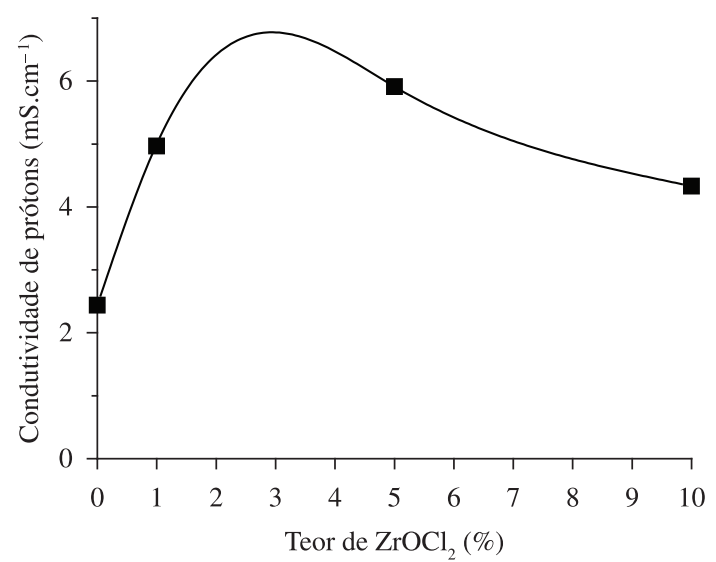

Figura 9. Condutividade protônica $(\sigma)$ de membranas SPEEK/CF preparadas com diferentes teores de cloreto de zirconila.

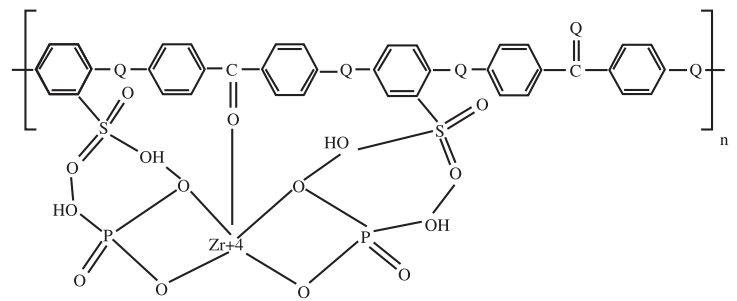

Figura 10. Figura esquemática mostrando as ligações de hidrogênio entre o ZrP e SPEEK ${ }^{[38]}$.

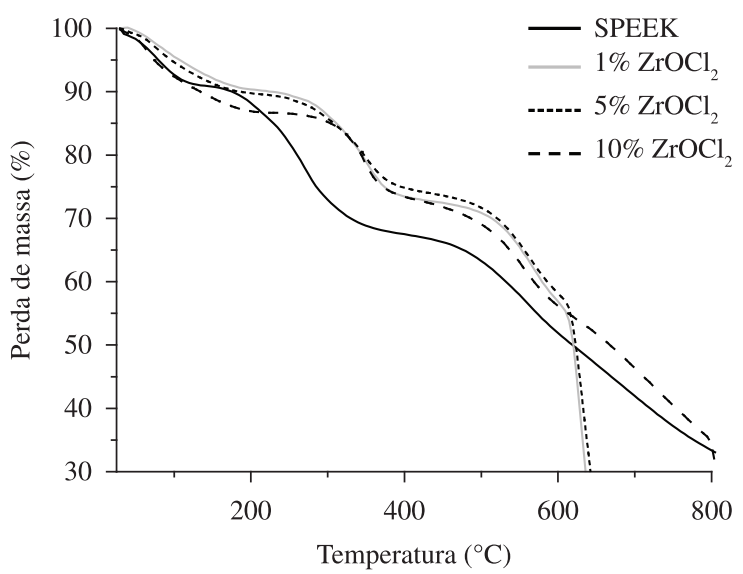

Figura 11. Curvas termogravimétricas das membranas híbridas SPEEK/CP zirconizadas com diferentes teores de cloreto de zirconila.

\section{Conclusões}

Membranas híbridas zirconizadas com potencial para aplicação em célula combustível do tipo PEM foram preparadas com sucesso a partir de SPEEK, copolissilsesquioxano fosfonado (CF) e cloreto de zircônila. A síntese do CF através da hidrólise e condensação dos grupos etóxi para a formação de ligações siloxano foi eficaz e confirmada pela presença de uma banda no espectro de FTIR em $1019 \mathrm{~cm}^{-1}$. O processo de sulfonação para obtenção de SPEEK foi eficiente e o grau de sulfonação foi de $67 \%$ conforme análise elementar. A morfologia da membrana preparada com $1 \%$ de $\mathrm{ZrOCl}_{2}$ apresentou cavidades, com partículas de $\mathrm{ZrP}$ amorfas precipitadas em seu interior, evidenciando que a adesão entre as fases orgânica e inorgânica é baixa. Todas as membranas preparadas com cloreto de zirconila mostraram maior condutividade que o SPEEK nas mesmas condições, alcançando um valor máximo de $5,91 \mathrm{mS} / \mathrm{cm}$ para aquela contendo $5 \%$ de $\mathrm{ZrOCl}_{2}$. A membrana preparada com a adição de $\mathrm{ZrOCl}_{2} 10 \%$ apresentou melhor estabilidade térmica, provavelmente devido à maior complexação entre os grupos sulfônicos da cadeia SPEEK e os grupos fosfônicos do ZrP.

\section{Agradecimentos}

Os autores agradecem à CAPES-DS pela bolsa a K.R.A., ao CNPq pelas bolsas IC a G.P.B. e M.P., à Victrex pelas amostras de PEEK e ao PAP-UDESC pelo apoio financeiro.

\section{Referências Bibliográficas}

1. Grant, P. - Nature, 424,p.129 (2003). http://dx.doi.org/10.1038/424129a

2. Gottesfeld, S.; Zawodzinski, T. A.; Alkire, R. C.; Gerischer, H.; Kolb, D. M. \& Tobias,C. W. - Adv. Electrochem. Sci. Eng., 5, p.220 (1997).

3. Inzelt, G.; Pineri, M.; Schultze, J. W. \& Vorotybtsev, M. A. - Electrochim. Acta, 45, p.2403 (2000). http://dx.doi.org/10.1016/S0013-4686(00)00329-7

4. Akay, G. R. - "Development and characterization of Composite Proton Exchange Membranes for Fuel cell Applications", Tese de doutorado, Middle East Technical University (2008).

5. Kerres, J.; Cui, W.; \& Reichle, S. - J. Polym. Sci., 34,p.2421 (1996). http:// dx.doi.org/10.1002/(SICI)1099-0518(19960915)34:12<2421::AIDPOLA17>3.0.CO;2-A

6. Kerres, J.;Zhang,W.\&Cui,W.N.-J.Polym. Sci.,36,p.1441 (1998). http:// dx.doi.org/10.1002/(SICI)1099-0518(19980715)36:9<1441::AIDPOLA12>3.0.CO;2-4

7. Miyatake, K.; Chikashige,Y. \& Watanabe, M. - Macromolecules, 36 , p.9691 (2003). http://dx.doi.org/10.1021/ma0354521

8. Hill, M. L.; Kim, Y. S.; Einsla, B. R. \& McGrath, J. E. - J. Memb. Sci., 283, p.102 (2006). http://dx.doi.org/10.1016/j.memsci.2006.06.016

9. Kozawa, Y.; Suzuki, S.; Miyayama, M.; Okumiya, T. \& Traversa, E. - Solid State Ion., 181, p.348 (2010). http://dx.doi.org/10.1016/j. ssi.2009.12.017

10. Silva, V. S.; Ruffmann, B.; Silva, H.; Gallego, Y. A.; Mendes, A.; Madeira, L. M. \& Nunes, S. P. - J. Power Sour., 140, p.34 (2005). http:// dx.doi.org/10.1016/j.jpowsour.2004.08.004

11. Prado, L. A. S.; Wittich, H.; Schulte, K.; Goerik, G.; Vasil, M.; Garamus, R.; Willumeit, S.; Vetter, S.; Ruffmann, B. \& Nunes, S. P. J. - Polym. Sci. Polym. Phys., 42, p.567 (2003).

12. Alberti, G. \& Casciola, M. - Solid State Ion., 97, p.177 (1997). http:// dx.doi.org/10.1016/S0167-2738(97)00070-2

13. Gomes, D.; Buder, I. \& Nunes, S.P. - J. Polym. Sci. Polym. Phys., 44, p.2278 (2006). http://dx.doi.org/10.1002/polb.20868

14. Zaidi, S. M. J.; Mikhailenko, S. D.; Robertson, S. D.; Guiver, M. D. \& Kaliaguine, S. - J. Memb. Sci., 173, p.17 (2000). http://dx.doi. org/10.1016/S0376-7388(00)00345-8 
15. Ponce, M. L.; Prado, L.; Ruffmann, B.; Richau, K.; Mohr, R. \& Nunes, S. P. - J. Memb. Sci., 217, p.5 (2003). http://dx.doi.org/10.1016/S03767388(02)00309-5

16. Prado, L. A. S.; Goerigk, G.; Ponce, M. L.; Garamus, V. M.; Wittich, H.; Willumeit, R.; Schulte, K. \& Nunes, S. P. J. - Polym. Sci. Polym. Phys., 43, p.2981(2005).

17. Aowen, Z. \& Zhentao, Z. - J. Polym. Sci., 261, p.107 (2005).

18. Nunes, S. P.; Ruffmann, B.; Rikowski, E.; Vetter, S. \& Richau, K. - J. Membr. Sci., 203, p.215 (2002). http://dx.doi.org/10.1016/S03767388(02)00009-1

19. Alberti, G.; Constantino, U.; Casciola, M.; Ferroni, S.; Massinelli, L. \& Staiti, P. - Solid State Ion., 145, p.249 (2001). http://dx.doi. org/10.1016/S0167-2738(01)00948-1

20. Rodríguez-Castellón, E.; Jiménez-Jiménez, J.; Jiménez-López, A.; Maireles-Torres, P.; Ramos-Barrado, J. R.; Jones, D. J. \& Rozière, J. - Solid State Ion., 125, p.407 (1999). http://dx.doi.org/10.1016/ S0167-2738(99)00202-7

21. Kaliaguine, S.; Mikhaillenko, S. D.; Wang, K. P.; Xing, P.; Robertson, G. \& Guiver, M.- Catal. Today, 82, p.213 (2003). http://dx.doi. org/10.1016/S0920-5861(03)00235-9

22. Pezzin, S. H.; Stock, N. \& Nunes, S. P. - J. Memb. Sci., 325, p.559 (2008). http://dx.doi.org/10.1016/j.memsci.2008.08.010

23. Jiang, R.; Russel, H. \& Fenton, J. M. - J. Power Sour., 150, p.120 (2005). http://dx.doi.org/10.1016/j.jpowsour.2005.03.180

24. Nieuwenhuyse, P. V.; Legare, V. B.; Boisson, F.; Cassagnau, P. \& Michel, A. - J. Non-Crystal. Solids, 354, p.1654 (2008). http://dx.doi. org/10.1016/j.jnoncrysol.2007.10.020

25. Sena, M. E.; Pinto, B. P. \& Santa Maria, L. C. - Mat. Lett., 61, p. 2540 (2007). http://dx.doi.org/10.1016/j.matlet.2006.09.060

26. Javaid, S. M. - Arabian J. Sci. Eng., 28, p.183 (2003).
27. Nakamura, H.; Nakamura, T.; Noguchi, T. \& Imagawa, K. - Polym. Degr. Stabil., 91, p.740 (2006). http://dx.doi.org/10.1016/j. polymdegradstab.2005.06.003

28. Xing, P.; Robertson, G. P.; Guiver, M. D.; Mikhailenko, S. D.; Wang, K. \& Kaliaguine, S. - J. Memb. Sci., 229, p.95 (2004). http://dx.doi. org/10.1016/j.memsci.2003.09.019

29. Muthu Lakshmi, R. T. S.; Choudhary, V. \& Varma, I. K. - J. Mater. Sci., 40, p.629 (2005). http://dx.doi.org/10.1007/s10853-005-6300-2

30. Saegusa, T. - Pure App. Chem., 67, p.1965 (1995).

31. Montoneri, E.; Viscardi, G.; Bottigliengo, S.; Gobetto, R.; Chierotti, M. R.; Buscaino, R. \& Quagliotto, P. - Chem. Mater., 19, p.2671 (2007). http://dx.doi.org/10.1021/cm063071r

32. Socrates, G. - "Infrared Characteristic Group Frequencies",Wiley, Chichester (1980).

33. Jones, D. J. \& Rozière, J. - J. Adv. Polym. Sci., 215, p.219 (2008).

34. Alberti, G. \& Casciola, M. - Annual Rev. Mat. Res., 33, p.129 (2003). http://dx.doi.org/10.1146/annurev.matsci.33.022702.154702

35. Kohli, P. \& Blanchard, G.J. - Langmuir, 16, p.695 (2000). http://dx.doi. org/10.1021/la990668v

36. Rikukawa, K. \& Sanui, K. - Prog. Polym. Sci., 25, p.1463 (2000). http://dx.doi.org/10.1016/S0079-6700(00)00032-0

37. Atwood, T. E.; Dawson, J. L.; Hoy, R. J.; Rose, J. B. \& Staniland, P. A. - Polym. Prep., Am. Chem. Soc., Div Polym. Chem., 20, p.191 (1979).

38. Sang, H.; Wu, Q. \& Huang, K. - J. Memb. Sci., 305, p.118 (2007). http://dx.doi.org/10.1016/j.memsci.2007.07.041

39. Krishna, P.; Park, J. S.;Yang, T. H.;Lee,Y. \& Kim, C. S. - Pow. Sources, 163, p.2 (2006). http://dx.doi.org/10.1016/j.jpowsour.2005.10.112

Enviado: 09/06/11 Reenviado: 23/12/11 Aceito: 02/01/12 